\title{
A Universal MIMO Approach for 3GPP Wireless Standards
}

\author{
Vasileios M. Kapinas and George K. Karagiannidis \\ Department of Electrical and Computer Engineering \\ Aristotle University of Thessaloniki \\ Thessaloniki, Greece \\ Email: \{kapinas, geokarag\}@auth.gr
}

\begin{abstract}
We propose a generalized framework for handling Multiple-Input Multiple-Output (MIMO) schemes defined within the context of the Third Generation Partnership Project (3GPP) physical layer specifications. A detailed review of all the multiantenna transmission techniques adopted by the 3GPP so far is firstly presented, starting from the Global System for Mobile (GSM) communications and passing through the High-Speed Packet Access (HSPA) releases to the very recent Long Term Evolution (LTE)-Advanced standard. Motivated by the outcome of this overview, we introduce the Transmit Matrix (TRAM) concept, that can include as special cases several MIMO systems (e.g. transmit diversity, spatial-multiplexing, etc.) regardless of the presence or not of a feedback channel. Our approach is based on the adoption of a novel measure called Necessary Transmit Information (NTI) and on its one-to-one correspondence with the TRAM. The last allows for a convenient alternative representation of a TRAM-based MIMO system model and serves the straightforward construction of NTI-codebooks for a wide range of transmission schemes. The indicative applications provided reveal the universal nature of the proposed methodology.
\end{abstract}

\section{INTRODUCTION}

Mobile broadband communications experience today great success with major standardization bodies, among them the Third Generation Partnership Project (3GPP), leading a continuous effort for the definition of the next-generation radio access technologies. Recently, the Long Term Evolution (LTE)Advanced has been approved by the International Telecommunication Union-Radiocommunication Sector (ITU-R) as a real fourth-generation (4G) technology and as such it has been part of the International Mobile Telecommunications (IMT)Advanced family of radio interfaces [1]. In the meantime, all LTE-Advanced predecessors, namely Global System for Mobile (GSM) communications, Enhanced Data rates for GSM (or Global) Evolution (EDGE), Wideband Code Division Multiple Access (WCDMA), High-Speed Packet Access (HSPA) and LTE, had already been included in the IMT-2000 family of technologies for third-generation (3G) mobile services [2]. A common characteristic among all these standards is the provision for Multiple-Input Multiple-Output (MIMO) techniques as a means to enhance system performance in terms of peak data rate and average spectral efficiency.

In this paper, we firstly provide a concise description of the single-user (MIMO) techniques included in the PHYsical layer (PHY) specifications of 3GPP standards. According to the authors' knowledge, no such a compact review gathering all the basic PHY details of the involved techniques exist in the literature. The outcome of this effort reveals that the MIMO technologies adopted by 3 GPP so far are mainly based on fundamental transmit/receive diversity and spatial multiplexing designs. This observation motivated us to establish a universal way for representing several transmission schemes that fall into these categories. To this end, we propose the Transmit Matrix (TRAM) concept, a quite arbitrary algebraic structure, and the Necessary Transmit Information (NTI) that conveys all the critical information required for the reconstruction of the TRAM. This becomes feasible through the one-to-one correspondence that holds between the TRAM and the NTI. We further proceed with the establishment of a TRAM-based MIMO system model that can be efficiently utilized for the implementation of different adopted decoding strategies. It is worth mentioning that, in the case of closed-loop MIMO systems, the associated precoder matrices can be embedded into the TRAM structure, thus allowing for the design of more compact codebooks based only on the NTI.

Decoding (MLD) or Maximum-Likelihood and closed-form results are derived for the linear processed received transmit physical antennas is

We point out that in the context of this paper we refer to MIMO in the wider sense, in contrast to the 3GPP glossary where MIMO implies only the multi-layer Spatial Multiplexing (SM) technique and its variants. Additionally, although we focus on single-user systems, the analysis adopted here can also be applied to multi-user schemes or to systems supporting similar advanced multi-antenna concepts such as the Coordinated Multi-Point (CoMP) transmission and reception at multiple geographically separated sites, aimed to be included in the forthcoming LTE-Advanced Release 11.

Notations: In the sequel, $*, T, \dagger$ denote complex conjugation, matrix transpose and Hermitian transpose. Furthermore, $\otimes, \mathbb{E}, \dot{\vee}$ define the Kronecker product, expectation and (set) exclusive disjunction, respectively.

\section{Multi-Antenna Configurations in 3GPP WIRELESS STANDARDS}

\section{A. $G S M / E D G E$}

Multi-antenna technology has been chosen among others by $3 \mathrm{GPP}$ as a means to define the evolutionary path of the first digital cellular system, namely GSM, towards higher data 
rates. To this end, General Packet Radio Service (GPRS), EDGE and their further enhancement under the combined GSM/EDGE Radio Access Network (GERAN), brought a variety of new features in the air interface of GSM. However, it was the EDGE Phase II (EDGE2) of Evolved GERAN (starting with Release 7) that ensured the future competitiveness of GSM in the international arena of wireless standards through the introduction of advanced components, such as the Mobile Station Receive Diversity (MSRD).

In more detail, MSRD is characterized by the Downlink Advanced Receiver Performance (DARP) Phase II requirements and can be considered as an extension of the Single Antenna Interference Cancellation (SAIC) already introduced in GERAN Release 6. The concept of utilizing 2 receive $(\mathrm{Rx})$ antennas at the base station (Base Station System or BSS) for achieving interference suppression and/or diversity gain had already been adopted by several GSM networks in order to improve the uplink (UL) performance. With MSRD this functionality was extended to the downlink (DL) through the provision of dual-antenna mobile terminals (Mobile Stations or MS) that have been proved to provide better interference handling and coverage improvement [3]. Evolved GERAN Releases 8-10 also support the use of $1 \times 2$ configurations for the transmission of both the DL and UL data channels (Packet Data Channels or PDCH) [4].

\section{B. WCDMA/HSPA}

Even from WCDMA or Universal Mobile Telecommunications System (UMTS) Release 99, dual-antenna transmission has been employed in the DL communication between the base station (Node B or NB) and the mobile terminal (User Equipment or UE) for operation in two modes, namely Frequency-Division Duplex (FDD) and Time-Division Duplex (TDD) [5]. Specifically, in the description of the Universal Terrestrial Radio Access (UTRA) Layer 1, two open-loop (OL) transmit (Tx) Diversity (TxD) schemes are defined; SpaceTime Transmit Diversity (STTD) for FDD or Space Code Transmit Diversity (SCTD) for TDD, and Time-Switched Transmit Diversity (TSTD) for both FDD and TDD. Closedloop (CL) Transmit Adaptive Array (TxAA) can also be used for FDD, whereas for TDD operation possible CL implementations include the TxAA and Selective Transmit Diversity (STD) schemes.

However, it was the Evolved HSPA (HSPA+) of Release 7 and beyond that pushed the peak data rates and cell capacity of DL HSPA (HSDPA) much higher through the introduction of the $2 \times 2$ Double-TxAA (D-TxAA) which extends the singlestream TxAA to dual-codeword CL SM. In HSPA+ Release 8, D-TxAA is combined with higher order modulation (64QAM) and also a new dual-layer CL SM scheme called PerAntenna Rate Control (PARC) is adopted for the low chip rate TDD option (1.28 Mcps TDD). In HSPA+ Release 9, DualCarrier HSDPA (DC-HSDPA) enables D-TxAA to operate simultaneously on two carriers of not necessarily contiguous frequency bands.
Due to the full backward compatibility of HSPA with the subsequent 25-series of specifications, all previous $2 \times 1$ and $2 \times 2$ schemes are supported as well in HSPA+ Release 10 [6], [7] for the transmission of the DL data channel (High Speed Physical Downlink Shared Channel or HS-PDSCH). ${ }^{1}$ In the same Release, 4-carrier HSDPA can use D-TxAA over the aggregated bandwidth of $20 \mathrm{MHz}$ (spread across one or two frequency bands). HSPA evolution continues with the forthcoming Release 11 specifications that provision for combination of $2 \times 2$ and $2 \times 4$ MIMO together with 8 -carrier HSDPA operation [8].

Concerning the UL data channel (Enhanced Dedicated Physical Data Channel or E-DPDCH), single-antenna transmission with 2-Rx diversity after proper combining at the NB is specified in HSPA+ Release 7, while HSPA+ Release 8 and beyond support the use of $2 \mathrm{Tx}$ antennas at the UE for timeswitched transmission. Finally, in HSPA+ Release 11, novel OL/CL TxD techniques for $2 \mathrm{Tx}$ antennas (e.g. beamforming) as well as dual-stream MIMO operation with 2 and $4 \mathrm{Rx}$ antennas at the NB are under study for possible inclusion [8].

\section{Long Term Evolution (LTE)}

According to the PHY description of the Evolved UTRA Network (E-UTRAN) [9], the base station (evolved Node B or eNB) can use 1, 2 or 4 Radio-Frequency (RF) chains for Orthogonal Frequency-Division Multiplexing (OFDM) transmission of the DL data channel (Physical Downlink Shared Channel or PDSCH), while the UE utilizes generally up to 4 antennas for reception. The possible multi-antenna configurations are $1 \times 2,2 \times 2,4 \times 2$ and $4 \times 4$. For the Physical Uplink Shared Channel (PUSCH), the Single-Carrier Frequency Division Multiple Access (SC-FDMA) technology has been adopted, while the UE can optionally support (1 out of 2 ) Tx antenna selection to achieve diversity gain without extra hardware complexity and prohibitive power consumption [10]. Reliable UL communication can be further achieved with additional Rx diversity gains through the deployment of 2 or 4 antennas at the eNB [11].

Some of the main single-user multi-antenna schemes supported in the DL of LTE Release 8 follow OL TxD. More precisely, for 2 Tx antennas, Space-Frequency Block Coding (SFBC) is implemented through the adoption of the Alamouti code [12] in the frequency domain, where the block coding process takes place over adjacent subcarriers instead of consecutive time-slots. For $4 \mathrm{Tx}$ antennas this technique is combined with Frequency-Switched Transmit Diversity (FSTD). The same diversity techniques are also utilized in the transmission of the Physical HARQ (Hybrid Automatic Repeat-reQuest) Indicator Channel (PHICH), with two types being available for the case of $4 \mathrm{Tx}$ antennas. Single-Input Multiple-Output (SIMO) configurations achieving at least $2 \mathrm{Rx}$ diversity at the $\mathrm{UE}$ are also provisioned for the DL.

\footnotetext{
${ }^{1}$ TSTD is an exception since for the FDD mode it is intended exclusively for the Synchronization Channel $(\mathrm{SCH})$ while for the 3.84 and 7.68 Mcps TDD options only for the SCH and the Secondary Common Control Physical Channel (S-CCPCH).
} 
The rest of DL MIMO schemes are associated with OL and CL multi-layer SM. Specifically, one or two codewords can be transmitted simultaneously using up to four layers (for the case of $4 \mathrm{Tx}$ and $4 \mathrm{Rx}$ antennas) after CL codebookbased precoding at the eNB relying on Cell-specific Reference Signals (CRS). A multiplexing-diversity tradeoff solution for 2 and $4 \mathrm{Tx}$ antennas is also provided by means of an OL SM scheme combined with large-delay Cyclic Delay Diversity (CDD), also called random beamforming [13]. This technique supports at least two layers and still relies on CRS and a predefined set of matrices (codebook) for precoding, though the applied precoders are a priori known to the UE. Finally, single-layer beamforming based on precoded UE-specific or Demodulation Reference Signals (DM-RS) constitutes another available option for the PDSCH transmission.

In LTE Release 9, dual-layer beamforming was introduced [14]. This transmission scheme is based on non-codebookbased precoder matrices and the use of two DM-RS, thus being considered as an extension of the single-layer beamforming already implemented in LTE Release 8. It is interesting to note that although non-codebook-based precoding is rather arbitrary and hence not visible in the LTE specifications, it can finally be reflected at the UE with the aid of the two precoded DM-RS [15]. Concerning the PUSCH transmission, no changes have been introduced in the PHY specifications.

\section{LTE-Advanced}

LTE-Advanced Release 10 was frozen in June 2011 with the possible use of advanced MIMO techniques being the key feature guaranteeing higher peak data rates and improved system capacity. The advances in MIMO technology have been accommodated with improvements in the reference-signal structure, comprised of enhanced DM-RS, newly introduced Channel-State Information Reference Signals (CSI-RS) and support of previous LTE CRS [16]. For the transmission of the PDSCH, all codebooks used for the precoding of SM with the CRS of LTE Releases 8 \& 9 can also be used along with the better designed CSI-RS. However, the maximum number of CSI-RS have now been extended to eight, thus making possible the simultaneous transmission of two codewords with up to eight layers through an $8 \times 8$ configuration. Specifications allow also for non-codebook-based precoding through extensive support of DM-RS for demodulation of up to eight layers [17], which can be seen as a direct evolution of the LTE Release 9 dual-layer beam-forming.

Undoubtedly, one of the most revolutionary enhancements in LTE-Advanced is the use of multiple antennas for the PUSCH transmission. According to [16], codebook-based precoded SM is now available for 2 and $4 \mathrm{Tx}$ antennas at the $\mathrm{UE}$, requiring at least equal number of $\mathrm{Rx}$ antennas at the eNB. Particularly, one or two transport blocks (or codewords) can be transmitted from the UE per UL component carrier. The modulation symbols associated with each of the transport blocks are mapped onto one to four layers according to the same principle as in DL SM [15]. However, the concept for the codebook design is much different; the prior Householder construction rule has been replaced by the Cubic Metric (CM) preserving constraint, and systematic UE Tx antenna selection is possible through dedicated precoders (selection vectors) [18]. We have to note that the possible multi-antenna configurations for the PUSCH transmission are $1 \times 2,1 \times 4$, $1 \times 8,2 \times 2,2 \times 4$ and $4 \times 4$.

Another UL multi-antenna configuration supported in LTEAdvanced is Tx diversity using 2 and 4 UE antennas, intended only for the transmission of the Physical Uplink Control Channel (PUCCH). This scheme is called Spatial OrthogonalResource Transmit Diversity (SORTD) and can provide more robust control signaling under poor (e.g. cell-edge) channel conditions. The basic principle of SORTD is to transmit the same control information from 2 different Tx antennas using orthogonal time-frequency resources [15], [18]. Assuming perfect orthogonality, the two signals can be received separately and combined constructively through Maximum Ratio Combining (MRC), thus providing twofold diversity. For the case of 4 Tx antennas, a transparent (or UE implementationspecific) scheme can be adopted to map the control signal and allocate the available resources to the desired antennas. A possible strategy could be to divide the $4 \mathrm{Tx}$ antennas into two pairs (e.g. according to their correlation) and allocate the same resources to antennas within a pair and orthogonal ones to antennas among different pairs. Another approach could be 2 out of 4 antenna selection [18].

\section{A UNIVERSAL MIMO APPROACH}

\section{A. Description of the Generic Multi-Antenna System}

We consider a closed-loop wireless communication system with $N_{t}$ physical antennas transmitting each a complex linear combination of modulation symbols which convey the information of one or more codewords (i.e. separate streams of channel coded bits) after being scrambled and mapped to an arbitrary two-dimensional constellation. The transmission is realized over successive time-slots, subcarriers or polarization modes, generally referred to here as Transmission Units (TU), while $N_{r} \mathrm{Rx}$ antennas combine the multipath replicas of the signals. Regardless of the TU type, each transmission takes place within a given Transmission Time Interval (TTI) as defined in the context of 3GPP specifications (e.g. for LTE, the TTI length is equal to a subframe of $1 \mathrm{~ms}$ ).

We assume that all antennas are sufficiently separated in space, frequency or polarization, such that the fades for each transmission path can be considered as statistically independent. Additionally, the possibility for flexible utilization of the frequency resources through the availability of very small bandwidth granularity, large number of subcarriers, subcarrier clustering within a single component carrier and carrieraggregation, can render realistic the hypotheses of sufficiently large channel coherence bandwidth and small Doppler spread. Under these considerations, the complex channel gain $h_{j i}$ from the $i^{\text {th }}$ transmit to the $j^{\text {th }}$ receive antenna remains constant for $N$ TUs before changing to a new realization, resulting in a random channel matrix $\mathbf{H} \in \mathbb{C}^{N_{r} \times N_{t}}$ with independent 
identically distributed (i.i.d.) complex entries and average fading power equal for all paths (flat power delay profile).

In more detail, every $N$ consecutive TUs, $n_{s}$ modulation symbols $\left\{s_{1}, \ldots, s_{n_{s}}\right\}$ are mapped into a matrix, which we call Transmit Matrix (TRAM) $\mathbf{S} \in \mathbb{C}^{N_{t} \times N}$, defining a block of length $l_{b}=N$ and efficiency $e_{b}=n_{s} / l_{b}$. The block (time) duration $t_{b}$ can be related to the TTI according to $t_{b}=k \times \mathrm{TTI}$, where the positive integer $k$ is equal to one for $\mathrm{TU}=\{$ subcarriers, polarization modes $\}$ or equal to $l_{b}$ for $\mathrm{TU}=$ time-slots $\}$. Obviously, the TRAM can be surjectively mapped to $\mathbf{s}=\left[s_{1} \cdots s_{n_{s}}\right]^{T}$, namely the symbol vector conveying all the block information. In the receiver side, full Channel State Information (CSI) is available, while part of this can be transferred back to the transmitter through a feedback channel to allow the transmitter adapt to the varying channel conditions.

\section{B. Algebraic Representation of the TRAM}

In order to allow the TRAM to be written in a compact algebraic form, we confine all entries located in the same column of $\mathbf{S}$ to take values within the set $\left\{s_{1}, \ldots, s_{n_{s}}\right\} \dot{\vee}\left\{s_{1}^{*}, \ldots, s_{n_{s}}^{*}\right\}$, implying that only symbols belonging to either the vector $\mathbf{S}$ or its conjugate $\mathbf{s}^{*}$ (but not together) can be linearly combined and transmitted from the active antennas per TU. This is the only restriction we impose on the design of $\mathbf{S}$ and fortunately this suits all MIMO cases that have already been included in the 3GPP standards. Therefore, the TRAM can be defined as

$$
\begin{aligned}
\mathbf{S} & =\mathbf{G} \Lambda_{S}^{\mathcal{C}}\left(\mathbf{I}_{N} \otimes \mathbf{s}\right) \quad \text { or } \\
\mathbf{S}^{T} & =\Lambda_{S}^{\mathcal{R}}\left(\mathbf{I}_{N} \otimes \mathbf{s}^{T}\right) \mathbf{G}^{T},
\end{aligned}
$$

where $\mathbf{I}_{N}$ is the identity matrix of order $N$ and $\mathbf{G}$ denotes a (column-wise) appended $N_{t} \times N n_{s}$ array consisting of the generally non-square submatrices $\mathbf{G}_{i} \in \mathbb{C}^{N_{t} \times n_{s}}, i=1,2, \ldots, N$, related to the coefficients of the $N$ TRAM columns. We refer to $\mathbf{G}$ as the Generator Matrix (GEM) of the TRAM.

The linear operator $\Lambda_{S}^{\mathcal{R}}$ (or $\Lambda_{S}^{\mathcal{C}}$ ) introduced in (2) (or (1)) is an endomorphism on $\mathbb{C}^{m \times n}$, with $m, n \in \mathbb{N}$, that induces complex conjugation on all or some of the $m$ (or $n$ ) matrix rows (or columns) according to the entries of the set $S$. Strictly speaking, $S \in \mathcal{P}\left(S_{\kappa}\right)$ where $S_{\kappa}=\{i: i \in \mathbb{N} ; i \leq \kappa\}$ and $\mathcal{P}\left(S_{\kappa}\right)$ is the power set of $S_{\kappa}$, while $\kappa$ stands generally for the number of rows $m$ or columns $n$. Obviously, $S$ can also be the empty set $\{\emptyset\}$ which in that case implies no conjugation. Additionally, if $\mathbf{A}, \mathbf{B} \in \mathbb{C}^{m \times n}$, then some basic properties of $\Lambda_{S}^{\mathcal{R}}, \Lambda_{S}^{\mathcal{C}}$, commonly denoted as $\Lambda_{S}^{\mathcal{R}, \mathcal{C}}$, follow as:

$$
\begin{aligned}
& \left(\Lambda_{S}^{\mathcal{R}, \mathcal{C}}(\mathbf{A})\right)^{\dagger, T}=\Lambda_{S}^{\mathcal{C}, \mathcal{R}}\left(\mathbf{A}^{\dagger, T}\right), \\
& \Lambda_{S}^{\mathcal{R}, \mathcal{C}}(\mathbf{A}+\mathbf{B})=\Lambda_{S}^{\mathcal{R}, \mathcal{C}}(\mathbf{A})+\Lambda_{S}^{\mathcal{R}, \mathcal{C}}(\mathbf{B}), \\
& \Lambda_{S}^{\mathcal{R}, \mathcal{C}}\left(\Lambda_{S}^{\mathcal{R}, \mathcal{C}}(\mathbf{A})\right)=\mathbf{A},
\end{aligned}
$$

The definition of these two operators can be extended to apply also on block matrices. In this case, we denote with $\Lambda_{S}^{\mathcal{R}_{N}}$ (or $\Lambda_{S}^{\mathcal{C}_{N}}$ ) the operation of complex conjugation on all or some of the $N$ row-wise stacked (or column-wise appended) blocks of submatrices according to the entries of the set $S$, where now $\kappa=N=m / \lambda$ (or $\kappa=N=n / \lambda$ ) with $\lambda \in \mathbb{N}$ being the number of rows (or columns) per submatrix. Finally, for the special case of a block diagonal matrix, the $\Lambda_{S}^{\mathcal{D}_{N}}$ notation will be preferred to denote complex conjugation on some or all of the $N$ submatrices of its main diagonal. The identities presented above will be used in Subsection III-C for the extraction of an alternative representation for a TRAMbased system model.

It is interesting to note that a given TRAM defines a unique triplet $(\mathbf{G}, S, N)$, hereafter referred to as Necessary Transmit Information (NTI), and vice versa, since all the structure information of the former is mapped to the latter in a distinct way. This one-to-one correspondence between TRAM and NTI is highly desired since the two operations of packing (TRAM $\mapsto \mathrm{NTI})$ and unpacking ( $\mathrm{NTI} \mapsto \mathrm{TRAM})$ are complementary and essential for the interactive communication between the Base Station (BS) and the Mobile Terminal (MT). Nevertheless, in order to give an insight on how the receiver could exploit the NTI and especially the GEM for demodulation purposes, we need to elaborate a little on the system model equations employing linear algebra processing.

\section{Equivalent System Model}

The complex baseband representation of the received matrix $\mathbf{R} \in \mathbb{C}^{N_{r} \times N}$ can be written as

$$
\mathbf{R}=\mathbf{H S}+\mathbf{N}
$$

where $\mathbf{N} \in \mathbb{C}^{N_{r} \times N}$ denotes the additive white noise matrix consisting of i.i.d. circularly-symmetric complex Gaussian random variables. For the sake of simplicity, we continue by examining each receive antenna separately. Thus, the system

$$
\mathbf{r}_{j}=\mathbf{S}^{T} \mathbf{h}_{j}+\mathbf{n}_{j}
$$

describes the Multiple-Input Single-Output (MISO) model associated with the $j^{\text {th }}$ receive antenna, where $\mathbf{r}_{j}, \mathbf{n}_{j} \in \mathbb{C}^{N}$ and $\mathbf{h}_{j} \in \mathbb{C}^{N_{t}}, j=1,2, \ldots, N_{r}$. By applying in both sides of (4) the operator $\Lambda_{S}^{\mathcal{R}}$ and after manipulations, we obtain

$$
\Lambda_{S}^{\mathcal{R}}\left(\mathbf{r}_{j}\right)=\mathbf{H}_{e, j} \mathbf{s}+\Lambda_{S}^{\mathcal{R}}\left(\mathbf{n}_{j}\right),
$$

where $\mathbf{H}_{e, j} \in \mathbb{C}^{N \times n_{s}}$ represents the Equivalent Channel Matrix (ECM) associated with the $j^{\text {th }}$ receive antenna, yielding

$$
\mathbf{H}_{e, j}=\Lambda_{S}^{\mathcal{R}}\left(\left(\mathbf{I}_{N} \otimes \mathbf{h}_{j}^{T}\right) \dot{\mathbf{G}}\right)
$$

It is important to note here that $\dot{\mathbf{G}}$ is the (row-wise) stacked $N N_{t} \times n_{s}$ array of all the GEM submatrices introduced in (1), namely if $\mathbf{G}=\left[\mathbf{G}_{1} \cdots \mathbf{G}_{N}\right]$ then $\dot{\mathbf{G}}=\left[\mathbf{G}_{1}^{T} \cdots \mathbf{G}_{N}^{T}\right]^{T}$.

From (6) it is obvious that the ECM structure depends exclusively on the triplet $(\mathbf{G}, S, N)$ and of course on the vector $\mathbf{h}_{j}$ that conveys all the instantaneous channels among the $N_{t}$ Tx and the $j^{\text {th }}$ receive antenna. This implies that given a full informed receiver in terms of both NTI and CSI, the most appropriate detection/equalization techniques can be selected among several options, including Linear Minimum Square Error (LMMSE) together with Successive Interference Cancellation (SIC) or Interference Rejection Combining (IRC) for 
both DL and UL and Maximum-Likelihood Decoding (MLD) or Maximum-Likelihood Sequence Estimation (MLSE) together with MRC for DL, among others. At the same time, it sounds proper to claim that the ECM inherits all its structure properties from the TRAM or equivalently that the NTI is transferred from the TRAM to the ECM. This verifies the intuition that an orthogonal TRAM would be highly desired, since from (5) it can be observed that any orthogonality in the ECM pattern would reduce (or even eliminate) the Intra-Block Interference (IBI) by means of (equivalent) channel-matched filtering. This is exactly the case for several DL schemes, such as the STTD, SCTD and TSTD in WCDMA/HSPA/HSPA+ and the OL TxD for 2 and 4 antennas in LTE/LTE-Advanced.

\section{IndiCATIVE APPLICATIONS \& DiscUSSION}

\section{A. MLD/MLSE with MRC Reception of an Arbitrary TRAM}

From (5) and after channel-matched filtering, the received signal vector $\mathbf{r}_{m, j} \in \mathbb{C}^{n_{s}}$ can be straightforwardly written as

$$
\mathbf{r}_{m, j}=\mathbf{H}_{e, j}^{\dagger} \Lambda_{S}^{\mathcal{R}}\left(\mathbf{r}_{j}\right)=\mathbf{H}_{m, j} \mathbf{s}+\mathbf{n}_{m, j},
$$

where $\mathbf{n}_{m, j}=\mathbf{H}_{e, j}^{\dagger} \Lambda_{S}^{\mathcal{R}}\left(\mathbf{n}_{j}\right)$ is the noise vector at the matched filter output and $\mathbf{H}_{m, j}=\mathbf{H}_{e, j}^{\dagger} \mathbf{H}_{e, j}$ is a square (generally sparse) matrix of order $n_{s}$, derived with the aid of (6) as

$$
\mathbf{H}_{m, j}=\Lambda_{S}^{\mathcal{C}_{N}}\left(\dot{\mathbf{G}}^{\dagger}\right) \Lambda_{S}^{\mathcal{D}_{N}}\left(\mathbf{I}_{N} \otimes \mathbf{h}_{j}^{*} \mathbf{h}_{j}^{T}\right) \Lambda_{S}^{\mathcal{R}_{N}}(\dot{\mathbf{G}}) .
$$

Taking now into account all the receiver branches, the MRC is completed by superimposing the associated signal vectors

$$
\mathbf{r}_{m}=\sum_{j=1}^{N_{r}} \mathbf{r}_{m, j}=\sum_{j=1}^{N_{r}} \mathbf{H}_{e, j}^{\dagger} \Lambda_{S}^{\mathcal{R}}\left(\mathbf{r}_{j}\right)=\mathbf{H}_{m} \mathbf{s}+\mathbf{n}_{m},
$$

where $\mathbf{n}_{m}=\sum_{j=1}^{N_{r}} \mathbf{n}_{m, j}$ is the filtered noise vector and $\mathbf{H}_{m}=$ $\sum_{j=1}^{N_{r}} \mathbf{H}_{m, j}$ defines the $n_{s} \times n_{s}$ matrix, related to the total diversity gain (if any) achieved by the TRAM, represented by

$$
\mathbf{H}_{m}=\Lambda_{S}^{\mathcal{C}_{N}}\left(\dot{\mathbf{G}}^{\dagger}\right) \Lambda_{S}^{\mathcal{D}_{N}}\left(\mathbf{I}_{N} \otimes \sum_{j=1}^{N_{r}} \mathbf{h}_{j}^{*} \mathbf{h}_{j}^{T}\right) \Lambda_{S}^{\mathcal{R}_{N}}(\dot{\mathbf{G}}) .
$$

Additionally, from (9) and making use of (5) and (6), we easily obtain the required linear processing at the receiver for MLD

$$
\mathbf{r}_{m}=\sum_{j=1}^{N_{r}} \Lambda_{S}^{\mathcal{C}}\left(\dot{\mathbf{G}}^{\dagger}\left(\mathbf{I}_{N} \otimes \mathbf{h}_{j}^{*}\right)\right) \Lambda_{S}^{\mathcal{R}}\left(\mathbf{r}_{j}\right) .
$$

A simple observation of (10) can easily lead to the conclusion that the TRAM structure affects the orthogonality of $\mathbf{H}_{m}$ and therefore the decoding complexity through the NTI. This can be easily realized if we consider that we have designed the TRAM in such a way that the resulted $\mathbf{H}_{m}$ is orthogonal. In this ideal case the IBI is zero and the MLD can be performed in a single-symbol way. However, in practice, the presence of non-trivial IBI at the receiver leads to the adoption of the more general symbol group-wise MLD (aka MLSE). Hence, if $n$ is the number of independently decoded symbol groups with $n \leq n_{s}$ and $g=1,2, \ldots, n$, the decoder can separately obtain an ML estimate of the symbol vector $\mathbf{s}_{g} \in \mathbb{C}^{n_{s} / n}$ via

$$
\hat{\mathbf{s}}_{g}=\underset{\mathbf{s}_{g} \in \mathcal{S}}{\arg \min }\left\|\mathbf{r}_{m}^{g}-\mathbf{H}_{m}^{g} \mathbf{s}_{g}\right\|^{2},
$$

where $\mathbf{r}_{m}^{g} \in \mathbb{C}^{n_{s} / n}$ is the associated received signal subvector, $\mathbf{H}_{m}^{g}$ is a square matrix of order $n_{s} / n$ derived from $\mathbf{H}_{m}$ after row/column elimination and $\mathcal{S}$ is the signal space under search.

In order to reveal the flexibility of the derived results and especially the universal nature of the TRAM-based approach, we proceed with the error performance comparison of four different transmission schemes, namely SIMO, STTD, Double STTD (DSTTD) [19] and OL SM [20] with 1,2, 4 and 2 Tx antennas, considering 2 and $4 \mathrm{Rx}$ branches for all cases. For the sake of simplicity, all TRAMs associated with these schemes refer to time-based TUs. However, similar results can be extracted for the case of systems defined in the frequency domain or involving several polarization modes. Additionally, closed-loop MIMO schemes and systems employing different reception strategies (e.g. [21]) can efficiently be handled by utilizing the same framework. Finally, we would like to note that the closed-form type of results derived in this paper can be promising tools for the analytic performance analysis of generic MIMO systems.

In Fig. 1, the average bit error probability $P_{b}$ of the above multi-antenna schemes are plotted versus the average Signalto-Noise Ratio (SNR) per bit and per receiver branch $\bar{\gamma}_{b}$ in $\mathrm{dB}$. The transmission is considered over a flat fading Ricean channel with factor $K=5 \mathrm{~dB}$ at a spectral efficiency of 6 bits per second per Hertz (bps/Hz) with 8-PSK and 64-QAM Graybased modulation mappings considered for the DSTTD, SM and SIMO, STTD schemes, respectively. The SNR is given by $\bar{\gamma}_{b}=\Omega \frac{N_{t} E_{s}}{\log _{2}(M) N_{0}}$, where $\Omega$ is the average fading power, $N_{0}$ the complex noise variance, $E_{s}=\mathbb{E}\left[\mathbf{s}^{\dagger} \mathbf{s}\right] / n_{s}$ the average symbol energy, and $M$ the cardinality of the constellation(s).

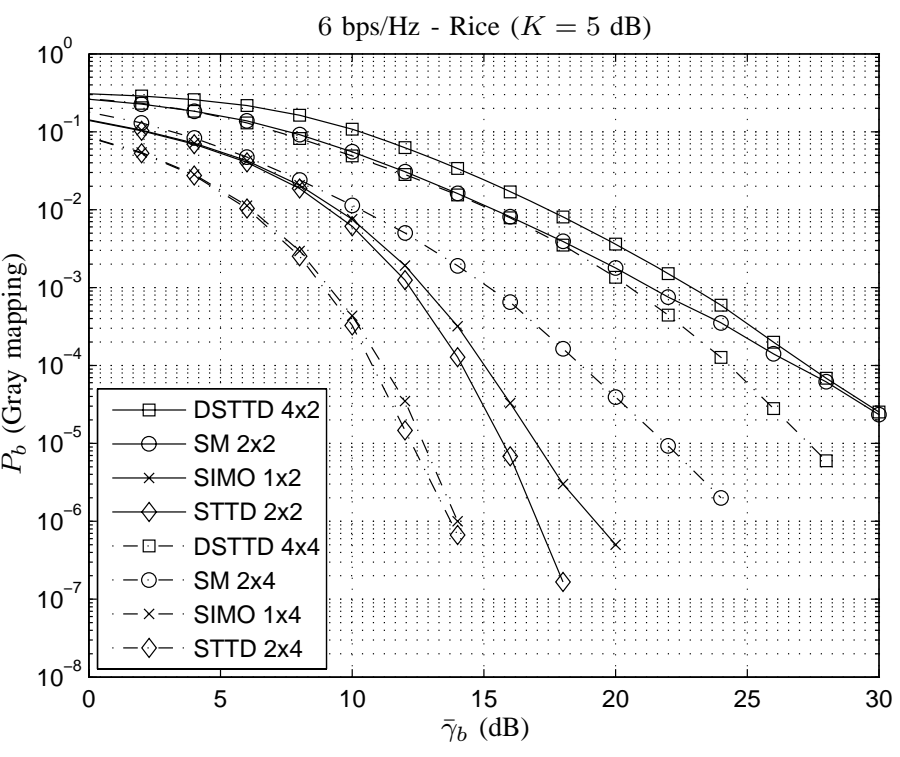

Fig. 1. Bit Error Rate (BER) performance of different MIMO schemes assuming MLD/MLSE with MRC over 2 and 4 antennas at the receiver. 


\section{B. NTI-based codebook for 2 Tx physical antennas}

In this section, we construct a unified NTI-based codebook for the case of two physical Tx antennas, serving the UE scheduling in the DL direction of LTE and LTE-Advanced networks. To begin with, the supported schemes can transmit modulation symbols associated with $d$ codewords using $n_{s}$ layers (denoted as $v$ in the specifications) per TTI, with $d \in\{1,2\}$ and $d \leq n_{s} \leq N_{t}$. We therefore assume that the information symbols involved per TRAM length can be associated with one or two codewords. The active antenna ports $P$ per TU generally follow the rule $P \leq N_{t}$, thus implying in our case that $P \in\{1,2\}$. We remind that the NTI is represented by the triplet $(\mathbf{G}, S, N)$, where $\mathbf{G}$ is the GEM of dimensions $N_{t} \times N n_{s}, S$ is the set including the indices of the conjugated columns in the TRAM matrix $\mathbf{S}$ and $N$ is the number of TUs (i.e. the length) of the TRAM.

For $P=1$, the single antenna transmission scheme is employed, for which it obviously holds that $n_{s}=1$ with the symbol vector given by $\mathbf{s}=\left[s_{1}\right]$. The $S, N$ parameters of the NTI take the values $S=\{\emptyset\}$ and $N=1$, while the GEM can be written as $\mathbf{G}=\left[\begin{array}{ll}1 & 0\end{array}\right]^{T}$ (or $\mathbf{G}=\left[\begin{array}{ll}0 & 1\end{array}\right]^{T}$ if we want to distinguish between the two antenna ports). Therefore, the TRAM reduces to the trivial form $\mathbf{S}=\left[\begin{array}{ll}s_{1} & 0\end{array}\right]^{T}$ and we write

$$
\mathbf{S}=\left(\begin{array}{c}
s_{1} \\
0
\end{array}\right) \sim \mathbf{G}=\left(\begin{array}{l}
1 \\
0
\end{array}\right), S=\{\emptyset\}, N=1 .
$$

For $P=2$, two fundamental schemes are supported, namely open-loop Tx diversity and closed-loop SM. For the former, the number of layers is equal to the number of $\mathrm{Tx}$ antennas and the TRAM can be reproduced from [16] as

$$
\mathbf{S}=\frac{1}{\sqrt{2}}\left(\begin{array}{cc}
s_{1} & -s_{2}^{*} \\
s_{2} & s_{1}^{*}
\end{array}\right)=\mathbf{G} \Lambda_{\{2\}}^{\mathcal{C}}\left(\mathbf{I}_{2} \otimes \mathbf{s}\right),
$$

which can be represented by the NTI as the triplet

$$
\mathbf{G}=\frac{1}{\sqrt{2}}\left(\begin{array}{cccc}
1 & 0 & 0 & -1 \\
0 & 1 & 1 & 0
\end{array}\right), S=\{2\}, N=2 .
$$

Concerning the closed-loop SM schemes, one or two layers may be used for transmission, defining the symbol vectors as $\mathbf{s}=\left[s_{1}\right]$ or $\mathbf{s}=\left[\begin{array}{ll}s_{1} & s_{2}\end{array}\right]^{T}$. In both cases, for the TRAM NTI equivalence it holds that

$$
\mathbf{S}=W \mathbf{s} \sim \mathbf{G}=W, S=\{\emptyset\}, N=1,
$$

where $W$ stands for the $2 \times 1$ and $2 \times 2$ precoder matrices described in [16] (4 and 3 available options, respectively).

Therefore, we observe that equations (13)-(15) define nine different triplets $(\mathbf{G}, S, N)$ corresponding to an equal number of different TRAMs. Initially, the network scheduler can decide in favor of a triplet (e.g. according to cell traffic) and send its associated index to the UE together with the transmitted information data. The latter can utilize the NTI and the closed-form results derived in the previous sections in order to combine and decode the received signals. On the other way, the UE can estimate the channel conditions experienced on its side and based on this to send or suggest a specific triplet from the NTI-based codebook to the eNB.

\section{ACKNOWLEDGMENT}

The authors would like to acknowledge the support provided by the Scientific Computing Center at A.U.Th. throughout the progress of this research work. They are also thankful to $\mathrm{Mr}$. Theodoros Chronis, researcher at the Aristotle University of Thessaloniki, for his fruitful cooperation.

\section{REFERENCES}

[1] ITU, Requirements related to technical performance for IMT-Advanced radio interface(s), ITU-R Rec. M.2134, Nov. 2008.

[2] — Detailed specifications of the terrestrial radio interfaces of International Mobile Telecommunications-2000 (IMT-2000), ITU-R Rec. M.1457, Rev. 10, Jun. 2011.

[3] 3GPP, Feasibility study for evolved GSM/EDGE Radio Access Network (GERAN) (Release 7), 3GPP TR 45.912, Rev. 7.2.0, Feb. 2007.

[4] - Radio transmission and reception (Release 10), 3GPP TS 45.005, Rev. 10.1.0, May 2011.

[5] R. T. Derryberry, S. D. Gray, D. M. Ionescu, G. Mandyam, and B. Raghothaman, "Transmit diversity in 3G CDMA systems," IEEE Commun. Mag., vol. 40, no. 4, pp. 68-75, Apr. 2002.

[6] 3GPP, Physical channels and mapping of transport channels onto physical channels (FDD) (Release 10), 3GPP TS 25.211, Rev. 10.0.0, Sep. 2010.

[7] - Physical channels and mapping of transport channels onto physical channels (TDD) (Release 10), 3GPP TS 25.221, Rev. 10.0.0, Dec. 2010.

[8] —, Overview of 3GPP Release 11, Rev. 0.0.7, Jun. 2011.

[9] - Evolved Universal Terrestrial Radio Access (E-UTRA); Physical Channels and Modulation (Release 8), 3GPP TS 36.211, Rev. 8.9.0, Dec. 2009.

[10] J. Lee, J.-K. Han, and J. C. Zhang, "MIMO technologies in 3GPP LTE and LTE-advanced," EURASIP J. Wirel. Commun. Netw., 2009, art. no. 302092.

[11] D. Martín-Sacristán, J. F. Monserrat, J. Cabrejas-Peñuelas, D. Calabuig, S. Garrigas, and N. Cardona, "On the way towards fourth-generation mobile: 3GPP LTE and LTE-advanced," EURASIP J. Wirel. Commun. Netw., 2009, art. no. 354089.

[12] S. M. Alamouti, "A simple transmit diversity technique for wireless communications," IEEE J. Sel. Areas Commun., vol. 16, no. 8, pp. 14511458, Oct. 1998.

[13] Q. Li, G. Li, W. Lee, M. -il Lee, D. Mazzarese, B. Clerckx, and Z. Li, "MIMO techniques in WiMAX and LTE: A feature overview," IEEE Commun. Mag., vol. 48, no. 5, pp. 86-92, May 2010.

[14] 3GPP, Evolved Universal Terrestrial Radio Access (E-UTRA); Physical Channels and Modulation (Release 9), 3GPP TS 36.211, Rev. 9.1.0, Mar. 2010.

[15] E. Dahlman, S. Parkvall, and J. Sköld, $4 G$ LTE/LTE-Advanced for Mobile Broadband. Oxford: Elsevier Ltd, 2011.

[16] 3GPP, Evolved Universal Terrestrial Radio Access (E-UTRA); Physical Channels and Modulation (Release 10), 3GPP TS 36.211, Rev. 10.2.0, Jun. 2011.

[17] S. Parkvall, A. Furuskär, and E. Dahlman, "Evolution of LTE toward IMT-advanced," IEEE Commun. Mag., vol. 49, no. 2, pp. 84-91, Feb. 2011.

[18] C. S. Park, Y.-P. E. Wang, G. Jöngren, and D. Hammarwall, "Evolution of uplink MIMO for LTE-advanced," IEEE Commun. Mag., vol. 49, no. 2, pp. 112-121, Feb. 2011.

[19] E. N. Onggosanusi, A. G. Dabak, and T. M. Schmidl, "High rate space-time block coded scheme: Performance and improvement in correlated fading channels," in Proc. IEEE Wireless Communications and Networking Conf., vol. 1, Orlando, FL, Mar. 17-21, 2002, pp. 194199.

[20] G. Foschini and M. Gans, "On limits of wireless communications in a fading environment when using multiple antennas," Wirel. Pers. Commun., vol. 6, no. 3, pp. 311-335, 1998.

[21] S. Morosi, E. D. Re, and L. Vettori, "Advanced receiver and MIMO schemes for LTE communications system," in Proc. 1st Int. Conf. Wireless Communication, Vehicular Technology, Information Theory and Aerospace and Electronic Systems Technology, Aalborg, Denmark, May 17-20, 2009, pp. 217-221. 